\title{
THE INITIAL PROGRESS OF NEW GENES WITH VARIOUS GENETIC SYSTEMS
}

\author{
W. F. BODMER and P. A. PARSONS \\ Genetics Department, University of Cambridge
}

\section{INTRODUCTION}

Received I.vi.6o

IN a diploid population, with two alleles $A$ and $a$ at a single locus, it was shown by Fisher (1922) that a stable equilibrium consisting of the 3 genotypes $A A, A a$ and $a a$ could be obtained if the viability of the heterozygote was greater than that of both homozygotes. $\mathrm{He}$ also showed that the gene frequency at the equilibrium point depended only on the viabilities of the genotypes. Several examples of such balanced polymorphisms have been examined in detail genetically, for example the hæmoglobin $A$ and $S$ genes (Allison, 1955), and the various inversions studied by Dobzhansky and his co-workers in Drosophila pseudo-obscura.

The theory of more complex situations has been examined recently. For example, Owen (I954) gave the conditions for stability for three alleles at a locus, and Mandel (1959a) has extended this problem to many alleles at a locus. Such analyses have obvious practical significance when we think for example of the $A B O$ blood groups, but, of course assume that adaptive values have remained constant throughout the course of the evolutionary process.

Another situation only recently examined is equilibria in autotetraploids. To treat this problem with complete generality is difficult, but by making some simplifications (Parsons, I959a), it is possible to derive the conditions for equilibrium. Bennett (I 957) and Mandel $($ I $959 b)$ have done analyses on sex-linked genes, and Owen (I953) has constructed an example where viabilities of genotypes differ between sexes.

In all these situations, to obtain stability, there must be some degree of heterozygote advantage and Owen (1959) has proposed a " heterozygosity principle" to generalise this.

The approach to population genetic problems, in which we determine the equilibrium points and their stability, has been used for many years. However, it is often of interest to consider the initial progress of a new gene or genotype rather than the progress near a stable equilibrium. The conditions for the establishment of a new gene may often be more important in the evolutionary history of a population than the conditions for a stable equilibrium. This approach to the study of population genetic models has been used by Bodmer ( $1960 a$ ) in the study of the population genetics of homostyle primroses and other problems (Bodmer, $1960 b$ ). As a simple illustration we 
will consider the classic example of random mating with two alleles at a single locus. If $p$ is the frequency of gene $A$ and $q$ the frequency of $a$, and the relative viabilities of the 3 genotypes $A A, A a$ and $a a$ are $a, h$ and $b$ respectively, such that amongst mature individuals they occur with relative frequencies $A A: A a: a a=a p^{2}: 2 h p q: b q^{2}$, then the gene frequency in the next generation is given by

$$
p^{1}=\frac{a p^{2}+h p q}{a p^{2}+2 h p q+b q^{2}}
$$

If, initially, a population only has gene $a$, and gene $A$ has been introduced by mutation, we can for the initial changes assume $p$ to be small and neglecting terms in $p^{2}$ equation (I.I) becomes

$$
p^{1}=\frac{h}{b} p
$$

This shows that for the introduced gene $A$ to increase in frequency we must have $h>b$. The heterozygote $A a$ must be superior in fitness to the original wild homozygote for the newly introduced gene $A$ to increase in frequency. Since the frequency of the homozygote $A A$ is of the second order in $p$, its viability does not affect the initial progress. The effective initial selective pressure is $(h / b)$, and if we put $h=\mathrm{I}$ and $b=\mathrm{I}-s$ where $s$ is small, this selective pressure is approximately $s$, since $\frac{h}{b}=\mathrm{I}+s$.

This case is clearly related to the conditions for a balanced polymorphism. The equilibrium frequency $p_{e}$ can be shown to be $\frac{h-b}{2 h-a-b}$ and for it to exist, $h-b$ and $h-a$ must both have the same sign. Now, since we have already shown that $h>b$ for an initial increase of $A$, $h>a$ as well. Such a system can be shown to be stable and is the example of a balanced polymorphism first analysed by Fisher (I 922 ). If $a>h>b$, then gene $A$ will not only increase as in the balanced polymorphism, but will completely replace the original gene $a$. In this and subsequent analyses we have ignored the small chance that a disadvantageous gene may increase in frequency in a small population, due to random sampling effects.

A consideration of the conditions under which a mutation will increase in a population is of obvious importance in population genetics. More complicated examples, such as those already mentioned, are amenable to analysis, since we can assume the gene frequency of the new mutant to be small. Also, the conditions for initial increase of a gene will, in some way, be related to the conditions for a stable equilibrium, as shown for the situation just analysed. Studying the conditions under which a new gene will increase in frequency should therefore give information which is both important and relatively easy to obtain for some rather complex population genetic models. 


\section{THE INTRODUCTION OF A THIRD ALLELE INTO A TWO ALLELE BALANCED POLYMORPHISM}

Following the notation of Owen (I954) and Mandel (I959a), we may write the viabilities of the genotypes formed with three alleles $A, B, C$ at a locus in the form

\begin{tabular}{l|ccc} 
& $A$ & $B$ & $C$ \\
\hline$A$ & $a$ & $h$ & $g$ \\
$B$ & $h$ & $b$ & $f$ \\
$C$ & $g$ & $f$ & $c$
\end{tabular}

The respective frequencies $p^{1}{ }_{1}, p^{1}{ }_{2}, p^{1}{ }_{3}$ of the three alleles $A, B$ and $C$ in one generation are given in terms of the frequencies $p_{1}, p_{2}, p_{3}$ in the preceding generation by the equations

where

$$
\begin{aligned}
& V p_{1}{ }_{1}=p_{1}\left(a p_{1}+h p_{2}+g p_{3}\right) \\
& V p^{1}{ }_{2}=p_{2}\left(h p_{1}+b p_{2}+f p_{3}\right) \\
& V p^{1}{ }_{3}=p_{3}\left(g p_{1}+f p_{2}+c p_{3}\right)
\end{aligned}
$$

$$
V=a p^{2}{ }_{1}+2 h p_{1} p_{2}+b p_{2}^{2}+2 g p_{1} p_{3}+2 f p_{2} p_{3}+c p^{2}{ }_{3} \text {. }
$$

Let us now suppose that the allele $C$ is introduced into a population containing the alleles $A, B$ in stable equilibrium. The frequencies of $A, B$, before the introduction of $C$ will then be (Fisher, I922)

$$
p=\frac{h-b}{2 h-a-b} \text { and } q=\mathbf{I}-p=\frac{h-a}{2 h-a-b} .
$$

We assume that initially $p_{3}$ is small, and that the frequencies of $A$ and $B$ after the introduction of $C$ depart from $p$ and $q$ by quantities of the same order of magnitude as $p_{3}$. Then from equation 2.I, on neglecting $p_{3}^{2}$ and higher powers, we have

$$
p_{3}^{1}=\frac{p_{3}(g p+f q)}{a p^{2}+2 h p q+b q^{2}}=k p_{3}
$$

where

$$
k=\frac{g p+f q}{a p^{2}+2 h p q+b q^{2}}
$$

Thus for $C$ to increase in frequency we must have $k>\mathrm{I}$. Substituting the values of $p$ and $q$ in the expression for $k$ we obtain

$$
k=\frac{g(h-b)+f(h-a)}{h^{2}-a b}=\frac{F+G}{-C}
$$

where $F=g h-a f, G=f h-b g$ and $C=a b-h^{2}$ are the cofactors of $f, g$ and $c$ in the viability determinant $\left|\begin{array}{ccc}a & h & g \\ h & b & f \\ g & f & c\end{array}\right|$. 
The condition that the allele $C$ should increase in frequency may therefore be written

$$
F+G+C>0 .
$$

By symmetry the corresponding conditions that $A$ should increase in the presence of $B$ and $C$ and that $B$ should increase in the presence of $A$ and $C$ are

$$
A+H+G>0 \text { and } H+B+F>0
$$

respectively where $A, B, H$ are the appropriate cofactors. It is interesting to note that the three conditions

and

$$
\begin{aligned}
& F+G+C>0 \\
& A+H+G>0 \\
& H+B+F>0
\end{aligned}
$$

together with the condition that it should be possible to have a balanced polymorphism with at least one pair of alleles on their own, is a necessary and sufficient condition for the existence of a stable equilibrium with all three alleles present (Mandel, I959a, p. 298). This means that if any allele $C$ can exist in stable equilibrium with two other alleles $A$ and $B$ at the same locus, then it must be possible for $C$ to increase in the presence of $A$ and $B$. The above conditions are easily generalised to deal with cases of more than three alleles at a locus.

Writing $h=\mathrm{I}$ and $a=\mathrm{I}-\alpha, b=\mathrm{I}-\beta$ the condition for the allele $C$ to increase in frequency becomes

$$
g \beta+f \alpha>\alpha+\beta-\alpha \beta .
$$

This expression does not involve $c$, the viability of the homozygote $C C$, which does not therefore affect the initial progress of the allele $C$. However different values of $c$ may determine whether $C$ replaces both $A$ and $B$, or only one of them, or remains in stable equilibrium with them both. When, for example $f, g>h$, a balanced polymorphism with all three alleles present is only achieved if $c<f$ and $g$. If $f>c>g$, $C$ replaces $A$ and we obtain a two allele balanced polymorphism with $C$ and $B$. However if $c>f, g, C$ replaces both $A$ and $B$. Given $a, h$ and $b$ the condition for $C$ to increase depends on the viabilities $f$, and $g$ of the new heterozygotes $B C$ and $A C$. The new allele $C$ will always increase in frequency when $f, g>h$ and when $f>h>g>a, b$. If $f>h, C$ will increase for any value of $g$ provided $f$ is sufficiently large. However if $h>f, g$ we must have both $f$, and $g>a, b$ for $C$ to increase but it will not necessarily increase in frequency if $h>f$, $g>a, b$.

Levene, Pavlovsky and Dobzhansky (1954) have studied experimental populations of Drosophila pseudo-obscura containing all three of the chromosome arrangements designated $S T, A R$ and $C H$. By considering the changes in frequency of the three types of arrangement, 
they were able to obtain estimates for the viabilities of all the possible genotypes that can be formed. These are shown in table 1 together with the necessary calculations to investigate completely the equilibrium and the various possible initial conditions. Since $F+G+C<0$, it is not possible with these viabilities (kept constant) for all three arrangements to co-exist in stable equilibrium, as was shown by Levene, Pavlovsky and Dobzhansky (1954). When $S T$ and $A R$ are present together in stable equilibrium, $\mathrm{CH}$ will not increase in frequency if introduced, for $\left|\frac{F+G}{C}\right|=0 \cdot 8680$ and is less than one. The frequency of $S T$ will increase extremely rapidly when introduced into a population in equilibrium for the arrangements $A R$ and $C H$, and should eventually completely replace $C H$ leaving a balanced polymorphism

TABLE I

An analysis of viability estimates for chromosome arrangement genotypes given by Levene, Pavlovsky and Dobzhansky (1954)

$$
\begin{array}{rlll}
S T / S T & 0.83(=a) & S T / A R & \mathrm{1} \cdot 00(=h) \\
A R / A R & 0.15(=b) & S T / C H & 0.77(=g) \\
C H / C H & 0.36(=c) & A R / C H & 0.62(=f) \\
A\left(=b c-f^{2}\right)=-0.3304 & B\left(=a c-g^{2}\right)=-0.2941 & C\left(=a b-h^{2}\right)=-0.8755 \\
F(=g h-a f)=0.2554 & G(=f h-b g)=0.5045 & H(=g f-h c)=0.1 \mathrm{I} 74 \\
F+G+C=-0.1156 & \left|\frac{F+G}{C}\right|=0.8680 \\
H+B+F=0.0787 & \left|\frac{H+F}{B}\right|=\mathrm{I} \cdot 2676 \\
A+H+G=0.2915 & \left|\frac{H+G}{A}\right|={ }_{\mathrm{I}} .8823
\end{array}
$$

with the two arrangements $S T$ and $A R$ only. It is not possible for the two arrangements $S T$ and $C H$ to exist together in stable equilibrium because the viability of the homozygote $S T / S T$ is higher than that of the heterozygote $S T / C H$.

In an earlier paper, Dobzhansky (1948) gave estimates of the viabilities obtained from experiments using two arrangements at a time which differ considerably from the estimates shown in table $\mathbf{I}$. Thus in a population with only $S T$ and $A R$ present, the viabilities of $S T / A R, S T / S T$ and $A R / A R$ are $\mathrm{I}, 0.8$ and 0.5 respectively. The condition that $C H$ should increase in frequency when introduced into such a population is

$$
0.5 g+0 \cdot 2 f>0 \cdot 6
$$

where, as before, $f$ and $g$ are the viabilities of the heterozygotes $A R / C H$ and $S T / C H$. The values of $f$ and $g$ required for $C H$ to increase in frequency are given in table 2. It is, of course, not possible for $\mathrm{CH}$ to increase in frequency when $f$ and $g$ take the values indicated by the triple experiment (table I). In order for $\mathrm{CH}$ to increase in frequency, 
at least the viability of $S T / C H$ and probably also that of $A R / C H$ must be higher in the initial stages when the frequency of $C H$ is still low. Similar conclusions apply to the introduction of $A R$ into a population in equilibrium for $S T$ and $C H$.

Changes in the adaptive values of the various genotypes have often been reported (see e.g. Dobzhansky, 1957) and it is clear that

TABLE 2

The values of $\mathrm{f}, \mathrm{g}$, the viabilities of $\mathrm{AR} / \mathrm{CH}$ and $\mathrm{ST} / \mathrm{CH}$, needed for $\mathrm{CH}$ to increase in frequency in the presence of $\mathrm{ST}$ and $\mathrm{AR}$

\begin{tabular}{|c|c|c|c|c|c|}
\hline$g=\quad \mathrm{I}$ & 0.9 & 0.8 & 0.77 & 0.7 & 0.6 \\
\hline$f>0.5$ & 0.75 & I & I 075 & $I \cdot 25$ & I. 5 \\
\hline
\end{tabular}

no model which assumes that the adaptive values remain constant can adequately explain the changes in frequency which have been observed in experimental populations. Levene, Pavlovsky and Dobzhansky (1954) found that the viabilities shown in table I estimated experimentally from the complete records, which extended over 365 days, did not give a satisfactory fit to the observed changes. They showed that estimates based only on the first 105 days of the records of the experimental populations differed significantly from the overall estimates, but they were not able to obtain satisfactory estimates for the latter part of the changes. An equilibrium containing about

\section{TABLE 3}

The limiting values of $\mathrm{f}$, and $\mathrm{g}$, the viabilities of $\mathrm{AR} / \mathrm{CH}$ and $\mathrm{ST} / \mathrm{CH}$, which just allow the presence of $\mathrm{CH}$ in stable equilibrium reith $\mathrm{ST}$ and $\mathrm{AR}$

\begin{tabular}{|c|c|c|c|c|c|}
\hline$g=$ & 0.77 & 0.8 & 0.83 & 0.85 & 0.9 \\
\hline$f>$ & I.3 & $I \cdot I 5$ & I $\cdot 00$ & 0.9 & 0.65 \\
\hline
\end{tabular}

80 per cent. ST, 5 per cent. $A R$ and 5 per cent. $C H$ is indicated by the observed data whereas all the viability estimates made on the data lead to the loss of the $C H$ arrangement. When the frequency of $C H$ is small its rate of increase is approximately $\frac{F+G}{-C}$ where $F, G$ and $C$ have the same meanings as before, and this must be greater than I for $C H$ to be able to co-exist with $A R$ and $S T$ in stable equilibrium. Only changes in the viabilities of the heterozygotes $S T / C H(=g)$ and $A R / C H(=f)$ are of any importance to the value of the quantity $F+G+C$ and hence the possibility of stable equilibrium with $C H$ present. Assuming the viabilities $a, b$ and $h$ are as in table I, $F+G+C=-0.8755+0.17 f-0.85 g$ so that the condition imposed on $f$ and $g$ for a stable equilibrium is $f>5\left(\mathrm{I}^{\circ} \mathrm{O}_{3}-g\right)$. The limiting values of $f$ and $g$ which would just allow the presence of $C H$ in a stable equilibrium are given in table 3 . 
The value of $g$ is the principal deciding factor and must, in general be increased in order that a stable equilibrium may be attained. Assuming that slight changes in the homozygote viabilities $b$ and $c$ have also occurred as indicated by Levene, Pavlovsky and Dobzhansky (I954) a reasonable fit to the observed equilibrium frequencies is provided by the set of viabilities

$$
\begin{array}{ll}
S T / S T(=a) 0 \cdot 8 & S T / A R(=h) \mathrm{I} \\
A R / A R(=b) 0 \cdot 2 & S T / C H(=g) 0 \cdot 9 \\
C H / C H(=c) 0 \cdot 3 & A R / C H(=f) 0 \cdot 7
\end{array}
$$

These give equilibrium frequencies

$S T: A R: C H=77 \cdot 78$ per cent. : $18 \cdot 5^{2}$ per cent. $: 3 \cdot 7$ per cent.

It seems possible that the viabilities of the heterozygotes $S T / C H$ and $A R / C H$ are near to the $S T / A R$ viability when their frequencies are low, but that the viabilities decrease as the population changes its constitution with increasing frequency of $C H$. However, the effect of selection during the further course of the evolutionary process may increase the viabilities of the inversion heterozygotes either by altering the genic contents of the inversions or by the selection of modifiers which interact with these genic contents. This may cause the viabilities to approach once again their original values and lead to a stable equilibrium containing all three arrangements.

\section{THE INTRODUCTION OF A NEW ALLELE INTO A TETRASOMIC POPULATION}

In tetrasomics, we must consider gametic genotype frequencies instead of gene frequencies. For two alleles $A$ and $a$, there will be three gametic genotypes $A A, A a$ and $a a$ whose respective frequencies may be denoted by $u, v$ and $w$ such that

$$
u+v+w=\text { I. }
$$

If the five possible zygotic genotypes, which can be denoted for brevity as $A_{4}, A_{3} a, A_{2} a_{2}, A a_{3}$ and $a_{4}$, have viabilities $a, b, c, d$ and $e$ respectively, the frequencies of the zygotes before and after selection are

Before selection

$A_{4}$
$A_{3} a$
$A_{2} a_{2}$
$A a_{3}$
$a_{4}$

Total

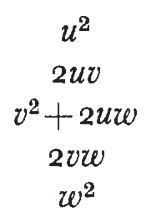

I
After selection

$$
\begin{gathered}
a u^{2} \\
2 b u v \\
c\left(v^{2}+2 u w\right) \\
2 d v w \\
e w w^{2} \\
T
\end{gathered}
$$

Now if the population is all $a_{4}$, and a mutation to $A$ occurs, then we can assume that $u, v$ are small and neglecting second order terms in $u$ and $v$,

$$
T=e+u(2 c-2 e)+v(2 d-2 e) .
$$


To this order of magnitude

$$
\begin{aligned}
& e u^{1}=\frac{1}{3} u c \\
& e v^{1}=\frac{4}{3} u c+d v
\end{aligned}
$$

where $u^{1}$ and $v^{1}$ are the gametic genotype frequencies in the next generation.

Here we have a somewhat more complex situation than the classical balanced polymorphism for 2 alleles at a diploid locus, since there are two parameters $u$ and $v$ about which we are attempting to make inferences. The 2 equations in $u$ and $v$ may be represented as a $2 \times 2$ matrix, and the latent roots derived. The magnitude of the dominant (largest) latent root $\lambda_{1}$, will determine whether or not the new gene will increase in frequency. For an increase in frequency $\lambda_{1}>$ I. The $2 \times 2$ matrix gives a characteristic equation

$$
\left(\frac{1}{3} c-\lambda e\right)(d-\lambda e)=0
$$

and when $\lambda_{1}>\mathrm{I}$, either $d>e$ and/or $c>3 e$. For an initial increase in frequency the former inequality is more likely to be important, and means that the fitness of the simplex genotype $A a_{3}>a_{4}$. There must therefore be a degree of heterozygous advantage. The latter relation, which is between the duplex and nulliplex genotypes implies that $A_{2} a_{2}>3 a_{4}$ or extreme heterosis, and will not usually be of great importance. The eventual situation in the population necessarily depends on the fitness of the other genotypes and, even for a simple case, computing the equilibrium values is not easy (Parsons r959a). The above conditions, however, are directly related to the conditions under which trivial equilibria, where say $A_{4}$ is absent, are stable. Parsons gives $d<e$ and $c<3 e$ as the conditions under which trivial equilibria are stable, or in the context of this paper, these are the conditions under which gene $A$ would not obtain a foothold in a population of $a_{4}$. These conditions can obviously be found by making $\lambda<\mathrm{I}$ in the above characteristic equation (3.3).

The complication of double reduction was ignored by Parsons (I 959a), but for a new mutant, it is not difficult to include this. If $\alpha$ is the coefficient of double reduction, it can be shown that

and

$$
\begin{aligned}
& e u^{1}=\frac{1}{3}(\mathrm{I}+2 \alpha) u c+\frac{1}{2} \alpha v d \\
& e v^{1}=\frac{4}{3}(\mathrm{I}-\alpha) u c+(\mathrm{I}-\alpha) v d
\end{aligned}
$$

The characteristic equation from the $2 \times 2$ matrix made up by these equations is

$$
\left[\frac{1}{3}(\mathrm{I}+2 \alpha) c-e \lambda\right][(\mathrm{I}-\alpha) d-e \lambda]-\frac{2}{3}(\mathrm{I}-\alpha) \alpha c d=0 .
$$

To examine the situation where $\alpha$ is small, let us write

$$
\lambda_{1}=\frac{d}{e}+\alpha x
$$

and

$$
\lambda_{2}=\frac{c}{3 e}+\alpha y
$$


the terms in $\alpha$ representing deviations from the roots at $\alpha=0$. Now putting $\lambda_{1}$ in the characteristic equation, and assuming terms in $\alpha^{2}$ to be negligible gives

and hence

$$
x=\frac{3^{d}(c-d)}{e\left(3^{d-c)}\right.}
$$

$$
\lambda_{1}=\frac{d}{e}\left[\mathrm{I}+\frac{3(c-d)}{3 d-c} \alpha\right] .
$$

To introduce the new gene $A, \lambda>\mathrm{r}$. Now if $c<d$, then

$$
\frac{3(c-d)}{3^{d-c}} \alpha<0
$$

and hence $d>e$ for the introduction of $A$, or that is $A a_{3}>a_{4}$ to introduce $A$ if $A a_{3}<A_{2} a_{2}$. However if $3 d>c>d$,

$$
\frac{3(c-d)}{3 d-c} \alpha>0,
$$

in which case it is possible for $d<e$, but $A$ increases in frequency. Thus we have a situation in which it is possible to introduce gene $A$, even though the heterozygous $A a_{3}$ genotype is less viable than $a_{4}$. Thus, heterozygote advantage itself becomes a condition which appears less necessary for the establishment of a gene in a tetraploid population when double reduction is taken into account, than for a polymorphism in a random mating diploid population. Without double reduction $d>e$, but with double reduction $d$ can be $<e$ for the new gene $A$ to increase in frequency. Double reduction, a meiotic phenomenon, leads to gametes being produced which contain sister alleles derived from the same chromosome, i.e. double reduction leads to a degree of homozygosis and in this sense is formally equivalent to a degree of inbreeding (Parsons, 1959b). With such a change of the breeding system it seems that heterozygote advantage as a prerequisite to establish a gene is no longer necessary. This type of conclusion will be further substantiated in the following section on mixed random mating and selfing in a diploid population.

The second latent root (3.8) will only be dominant if $c>3 d$. Following through an argument similar to that given for $\lambda_{1}$, it can be shown that for small $\alpha$

$$
\lambda_{2}=\frac{c}{3^{e}}\left[\mathrm{I}+\frac{2 c \alpha}{c-3^{d}}\right] .
$$

If $c>3 d$, then $c>3 e$ for $\lambda_{2}>\mathrm{I}$. Thus this latent root will only be of relevance when there is extreme heterosis, and as such is likely to be less important than $\lambda_{1}$.

To derive the conditions under which a gene will increase in frequency is therefore not difficult, due to the various approximations 
possible. In the establishment of equilibrium points, such approximations cannot usually be made, except when the stability of trivial equilibria in which one or more genotypes is wholly absent, is being investigated. Thus an explicit algebraic solution for the ultimate equilibrium points is often more difficult. To solve the problem of equilibria in auto-tetraploids, including the complication of double reduction, would probably need an electronic computor. Only by ignoring $\alpha$ and putting $a=e, b=d$ and $c=\mathrm{I}$, so creating a "symmetrical " of viabilities was it possible to obtain a tolerably simple algebraic solution (Parsons, 1959a).

\section{INTRODUCTION OF A NEW ALLELE WHEN THERE IS MIXED SELFING AND RANDOM MATING}

Another problem which shows the effects of the breeding system on the evolutionary progress of a gene is given by a population of plants reproducing partly by cross-fertilisation and partly by selfing. Many plants reproduce in such a manner, and only recently has the population genetics of some of these species been studied in any detail (for example, the lima bean (Allard, 1954) and the winter bean (Fyfe, 1952)). Hayman (1953) has developed a theoretical model in which equilibrium points were evaluated assuming the homozygotes to be at a disadvantage.

Let $s$ and $t$ be the proportion of selfing and random mating respectively such that $s+t=\mathrm{I}$. Let $u, v$ and $w$ be the frequencies of $A A, A a$ and $a a$ respectively such that $u+v+w=\mathrm{I}$ and let $a, h$ and $b$ be their respective viabilities. Under a mixture of random mating and selfing the genotypic frequencies will be:

$$
\begin{array}{cl}
\text { Genotype } & \multicolumn{1}{c}{\text { Frequency }} \\
A A & a\left[s\left(u+\frac{1}{4} v\right)+t\left(u+\frac{1}{2} v\right)^{2}\right] / T \\
A a & h\left[\frac{1}{2} s v+2 t\left(u+\frac{1}{2} v\right)\left(w+\frac{1}{2} v\right)\right] / T \\
a a & b\left[s\left(w+\frac{1}{4} v\right)+t\left(w+\frac{1}{2} v\right)^{2}\right] / T
\end{array}
$$

where $T$ is a divisor such that the total is unity. If $u^{1}, v^{1}$ and $w^{1}$ are the genotypic frequencies in the next generation, then

$$
\left.\begin{array}{l}
T u^{1}=a\left[s\left(u+\frac{1}{4} v\right)+t\left(u+\frac{1}{2} v\right)^{2}\right] \\
T v^{1}=h\left[\frac{1}{2} s v+2 t\left(u+\frac{1}{2} v\right)\left(w+\frac{1}{2} v\right)\right] \\
T w^{1}=b\left[s\left(w+\frac{1}{4} v\right)+t\left(w+\frac{1}{2} v\right)^{2}\right]
\end{array}\right\}
$$

If the population is initially all $a$ and a mutation occurred to $A$, then $u$ and $v$ are small. Neglecting quadratic terms in $u$ and $v$ and putting $w=\mathrm{I}-u-v$ gives

$$
\left.\begin{array}{l}
T u^{1}=a\left[s\left(u+\frac{1}{4} v\right)\right] \\
T v^{1}=h\left[\frac{1}{2} s v+2 t\left(u+\frac{1}{2} v\right)\right] \\
T w^{1}=b\left[s\left(\mathrm{I}-u-\frac{3}{4} v\right)+t(\mathrm{I}-2 u-v)\right]
\end{array}\right\}
$$


Hence

Thus

$$
T u^{1}=a\left[s\left(u+\frac{1}{4} v\right)\right]=u^{1}(b s+b t)=u^{1} b .
$$

$$
u^{1}=\frac{a s}{b} u+\frac{a s}{4 b} v
$$

Similarly

Thus

$$
T v^{1}=h\left[\frac{1}{2} s v+2 t\left(u+\frac{1}{2} v\right)\right]=v^{1} b .
$$

$$
v^{1}=\frac{2 h(\mathrm{I}-s)}{b} u+\frac{h\left(\mathrm{I}-\frac{1}{2} s\right)}{b} v
$$

The coefficients of $u$ and $v$ in these two equations form a $2 \times 2$ matrix, and for the conditions that $A$ will increase in the population we require its dominant (largest) latent root $\lambda_{1}>\mathrm{I}$.

The characteristic equation, from which $\lambda$ may be found is

$$
\left.(a s-\lambda b)\left[h\left(\mathrm{I}-\frac{1}{2} s\right)-\lambda b\right)\right]-\frac{a s h(\mathrm{I}-s)}{2}=0 .
$$

If we put $s=0$, that is we assume complete random mating, then $\lambda_{1}>\mathrm{I}$ if $h>b$ as derived in the introduction. Putting $s=\mathrm{I}$, or assuming complete selfing, for $\lambda_{1}>\mathrm{I}$ we must have

$$
\begin{array}{ll}
\text { either } & h>2 b \\
\text { or } & a>b .
\end{array}
$$

The first condition implies that the heterozygote $A a$ must have a viability over twice that of the original homozygote $a a$ for the successful introduction of $A$. The second condition implies that the viability of $A A>a a$ to establish gene $A$. In any particular problem inequality $\left(4^{\cdot 6}\right)$ or $\left(4^{\cdot} 7\right)$ will be the more important according to whether $h>2 a$ or $h<2 a$. For complete selfing $\left(4^{*} 7\right)$ is likely to be the more important, as $(4 \cdot 6)$ implies a considerable heterozygote advantage.

The quadratic equation (4.5) for $\lambda$ may be written

$$
\left.\lambda^{2} b^{2}-\lambda b\left[b\left(\mathrm{I}-\frac{1}{2} s\right)+a s\right)\right]+\frac{a s h}{2}=0
$$

and has roots

$$
\frac{b\left[h\left(\mathrm{I}-\frac{1}{2} s\right)+a s\right] \pm b \sqrt{a^{2} s^{2}+h^{2}\left(\mathrm{I}-\frac{1}{2} s\right)^{2}-a s^{2} h}}{2 b^{2}} .
$$

If now we put $h=\mathrm{I}$ and $a=\mathrm{I}+\alpha$, then on neglecting terms of order $\alpha^{2} s^{4}$, the two roots are

and

$$
\lambda_{1}=\frac{\mathrm{I}}{b}\left[\mathrm{I}+\frac{\alpha s}{2-s}\right]
$$

$$
\lambda_{2}=\frac{s}{b}\left[\frac{1}{2}+\frac{\alpha(\mathrm{I}-s)}{2-s}\right]
$$


We have

and this is positive if

$$
\lambda_{1}-\lambda_{2}=\frac{1}{b}\left[\mathrm{I}-\frac{1}{2} s+\frac{\alpha s^{2}}{2-s}\right],
$$

$$
\alpha>-\frac{(2-s)^{2}}{2 s^{2}} \text {. }
$$

The expression in $s$ is greatest when $s=\mathrm{I}$ so that provided $\alpha>-\frac{1}{2}$ or $a>h / 2, \lambda_{1}$ is the dominant latent root. In this case the condition for the new gene $A$ to increase is

If we write $b=\mathrm{I}+\beta$ this becomes

$$
\frac{\mathrm{I}}{b}\left[\mathrm{I}+\frac{\alpha s}{2-s}\right]>\mathrm{I} .
$$

$$
\beta<\frac{\alpha s}{2-s} .
$$

The condition (4.8) can never be satisfied when $\beta>0$ and $\alpha<0$, as is to be expected, for then the viabilities are in the order $A A<A a<a a$, and the new gene $A$ is at an unconditional disadvantage. The condition is always satisfied when $\beta<0$ and $\alpha>0$ and $A$ is at an unconditional advantage.

When $s$ is small (4.8) is approximately

$$
\beta<\frac{\alpha s}{2}
$$

For $\alpha<0$, this implies $\beta<0$, giving the usual heterotic or overdominant condition. However, when $\alpha>0$ it is possible for $A$ to increase even though $\beta>0$ or $h<b$, provided $\beta<\alpha s / 2$. For example, when $a=$ I I o per cent. and $s=20$ per cent. the viability of $a a$ must be less than ror per cent. of the viability of $A a$ for $A$ to increase. This effect, though small, implies that it is possible to have a situation in which gene $A$ becomes established even when there is no heterozygote advantage. This is in contrast to the situation when there is only random mating, for which heterozygote advantage is a prerequisite for a new gene to increase in frequency.

For values of $s$ near I when there is a high proportion of selfing, we can put $s=I-\sigma$ where $\sigma$ is small. The condition (4.8) is then

$$
\beta<\alpha(1-2 \sigma) \text {. }
$$

When $\alpha, \beta>0$ or $\alpha>0$ and $\beta<0$ this implies that $b<a$, that is the viability of the new homozygote $A A$ must be greater than that of the old homozygote $a a$. However if $\alpha, \beta<0,(4.9)$ may be written

$$
\sigma>\frac{|\alpha|-|\beta|}{2|\alpha|}
$$

where $|\alpha|$ and $|\beta|$ are the positive values of $\alpha$ and $\beta$. In this case it is possible for the new gene $A$ to increase even when $|\alpha|>|\beta|$ or $b>a$, provided $\sigma$, the amount of cross-fertilisation, is greater than 
the value specified by (4.10). Thus when there is mostly selfing the dominant condition for a new gene $A$ to increase is that the viability of $A A$ is greater than that of $a a$. However, this condition may be superseded by the overdominant state, with $A a$ superior to both $A A$ and $a a$, provided there is enough cross-fertilisation to produce an appreciable number of the heterozygotes $A a$.

When $s$ is near $\mathrm{I}$ and $a<h / 2$ then $\lambda_{2}$ becomes the dominant root. The condition for increase of $A, \lambda_{2}>\mathbf{I}$, is satisfied when $b<h / 2$, so

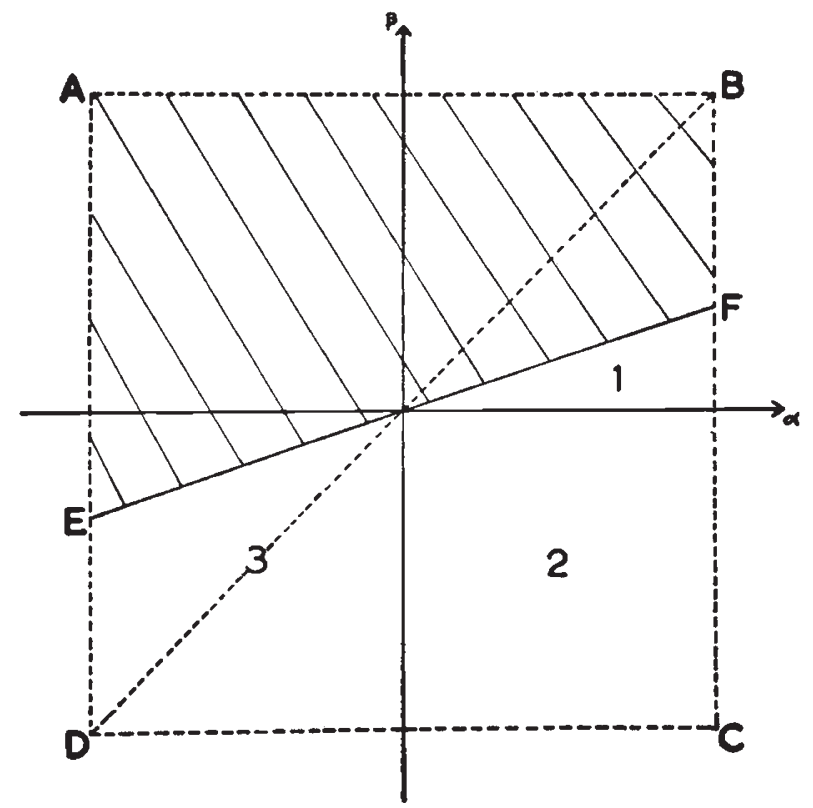

Fig. I.-The range of viabilities which allow for increase of a new allele under mixed random mating and selfing.

Only viabilities falling in the unhatched regions allow the new allele to increase.

$\mathrm{I}+\alpha, \mathrm{I}$, and $\mathrm{I}+\beta$ are the viabilities of the genotypes $A A, A a$ a $a$ respectively; $A B C D$ is the square enclosing all points for which $|\alpha|,|\beta|<\frac{1}{2}$

$$
E F \text { is the line } \beta=\frac{\alpha s}{(2-s)}
$$

(I) is a region of " negative heterosis" $\beta, \alpha>0$.

(2) is a region in which $A$ is at an unconditional advantage $\alpha>0, \beta<0$.

(3) is a region of "positive heterosis" $\beta, \alpha<0$.

that in this case the heterozygote $A a$ must be more than twice as fit as either homozygote.

The situation when $a>h / 2$ and $\lambda_{1}$ is the dominant root can be summarised diagrammatically as in fig. I. If we consider $\alpha$ and $\beta$ as Cartesian co-ordinates in a plane, the condition (4.8) implies that all points $(\alpha, \beta)$ lying below the line $\beta=\frac{\alpha s}{2-s}$ give values of the viabilities which will allow the new gene to increase in frequency. This is the unhatched region in the diagram, lying below the line $E F$ and inside the square $A B C D$ which encloses all values of $\alpha$ and $\beta$ 
less than $\frac{1}{2}$ in magnitude. There are three sub-regions characterised as follows:

(I) $\beta>0, \alpha>0$ is a region of " negative heterosis",

(2) $\beta<0, \alpha>0$ is a region in which $A$ is at an unconditional advantage,

(3) $\beta<0, \alpha<0$ is a region of "positive heterosis".

As $s$ increases from o to I the line $E F$ turns from a position along the $\alpha$-axis to one lying along $D B$. Thus, as the proportion of selfing increases, the area of the region (I) of negative heterosis increases while the area of the positive heterosis region (3) decreases. The region (2) in which $A$ is at an unconditional advantage remains unchanged.

If the frequency of genes produced by mutation were the same for all values of $\alpha$ and $\beta$, then the areas of these three regions would correspond to the expected frequencies of the three corresponding types of situations in natural populations. The area of region (2) $=\frac{1}{4}$ and that of $(\mathrm{I})=\frac{s}{8(2-s)}$, so that the area of $(3)=\frac{1}{4}-\frac{s}{8(2-s)}$. Thus the positive heterotic area is greater than the negative heterotic area and the ratio of the two areas decreases to I as $s$ increases to I. Clearly it is unrealistic to suppose that genes with different values of $\alpha$ and $\beta$ will be produced in equal frequency. If for example a majority of new heterozygotes were inferior to either homozygote $(\alpha, \beta>0)$, a situation which is not unlikely on the basis of recent studies of protein structure, a small amount of selfing might appreciably increase the proportion of non-heterotic genes to be found in a population.

It is unlikely that a detailed examination of assortative mating will lead to conclusions differing qualitatively from the above analysis of a mixture of random mating and selfing. Differing values of $s$, the proportion of selfing, will correspond to differing amounts of assortative mating. Thus, in conclusion, it may be said that whereas with random mating all genes which increase must have heterozygote advantage, increasing amounts of inbreeding allow for an increasing number of new genes which can gain a hold in the population without having any advantage in the heterozygote. Heterozygote advantage is clearly a product of the breeding system.

\section{DISCUSSION}

The initial conditions of progress for a newly occurring gene are clearly of critical importance to its future evolutionary history, and precede any conditions that may be necessary for its establishment in a balanced polymorphism. In this paper we have shown how the initial progress of a gene may be studied with relative ease for a number of complex genetic systems and how the conditions for it to increase may sometimes in fact, be related to the conditions for a 
stable equilibrium. Thus in the classic case of a two allele balanced polymorphism in a random mating diploid population, for a new gene $A$ to increase in frequency, the heterozygote $A a$ must be fitter than the prevailing homozygote $a a$, and this is part of the condition for the establishment of a balanced polymorphism. The viability of the homozygote $A A$ is not relevant to the initial progress under these conditions. Only when there is some inbreeding is this viability of importance and then an initial heterozygote advantage is no longer a prerequisite for the establishment of the new gene. These facts apply equally to all the genetic systems we have studied. Heterozygote advantage as observed in natural populations is clearly a product of the breeding system. Only those genes with some heterozygote advantage over the prevailing genotype will be found to occur with any appreciable frequency in a predominantly outbreeding population, because it is only such genes which can increase in frequency when first introduced into the population.

The classical models of population genetics assume that viabilities remain constant throughout an evolutionary process. However, Teissier and L'Héritier (I937) in their original studies on the change in frequency of the ebony gene in experimental populations of Drosophila suggested that selective values did not remain constant. They obtained evidence that the fecundity of an ebony female was reduced less in the presence of roo ebony females than in the presence of Ioo normal females, thus providing striking evidence for the dependence of the viability of a genotype in a population on the frequencies of co-existing genotypes. This phenomenon is also well illustrated by the classic work of Dobzhansky and his co-workers on the polymorphism for chromosome rearrangements in Drosophila pseudo-obscura. Thus Levene, Pavlovsky and Dobzhansky (I954) showed that their results for experimental populations containing the three arrangements $S T, A R$ and $C H$ could not easily be explained using a model with constant viabilities. In the second section of this paper we have suggested how their results could be explained by both environmental modification of selective values due to initial changes in the relative frequencies of the various genotypes, and selective modification of the viabilities in the later stages of the experiment, allowing the establishment of a balanced polymorphism.

Teissier (I954) has pointed out that two alleles could exist together stably in a random mating population without superiority of fitness of the heterozygote over both homozygotes, providing the heterozygote is always fitter than the predominant homozygote, but not necessarily fitter than the minority homozygote. This result follows from equation (1.2). It is analogous to a negative feedback system ensuring the maintenance of both alleles in a population. Clearly during the initial stages of progress of a new advantageous gene there will be little opportunity for the selective modification of viabilities, and a model assuming these to be constant will usually be quite valid. 
However the same validity by no means applies to the study of the stability of the possible equilibria formed with the new gene present.

The evidence available in favour of the possibility of selective modification of the heterozygote fitness during the course of an evolutionary change suggests an extension to Fisher's theory of the evolution of dominance (see e.g. Fisher (1958)). This is the evolution of overdominance. In other words the occurrence of an excess advantage of a heterozygote over both corresponding homozygotes may be due to an evolutionary process which has had more opportunity to favour consistently the heterozygote than either homozygote. This mechanism will be discussed in more detail in a further publication.

\section{SUMMARY}

I. The conditions for the establishment of a newly occurring gene in a population are discussed for three genetic systems; namely the introduction of a third allele into a two allele balanced polymorphism, a random mating tetrasomic population with and without double reduction, and a diploid population in which there is mixed selfing and random mating.

2. In an outbreeding population some heterozygote advantage is essential for a new gene to become established in a population, but with some inbreeding this is no longer a pre-requisite.

3. Available evidence appears to favour the possibility of the selective modification of the heterozygote fitness during the course of the establishment and spread of a new gene in an outbreeding population.

\section{REFERENCES}

Allard, R. W. 1954. Natural hybridisation in lima beans in California. Proc. Amer. Soc. Hort. Sci., 64, 410-416.

ALlison, A. C. 1955. Aspects of polymorphism in man. Cold Spring Harbor Symp. Quant. Biol., 20, 239-255.

BENNETT, J. H. 1957. Selectively balanced polymorphism at a sex-linked locus. Nature, 180, I363-1364.

Bodmer, W. F. I 960 a. The genetics of homostyly in populations of Primula vulgaris. Phil. Trans. Roy. Soc. B, 242, 51 7-549.

BODMER, W. F. I $960 b$. Discrete stochastic processes in population genetics. $\mathcal{F}$. Roy. Stat. Soc. B., 22, 21 8-244.

DOBZHANSKY, TH. 1948. Genetics of natural populations XVIII. Experiments on chromosomes of Drosophila pseudo-obscura from different geographic regions. Genetics, 33, 588-602.

DOBZHANSKy, TH. 1957. Mendelian populations as genetic systems. Cold Spring Harbor Symp. Quant. Biol., 22, 385-393.

FISHER, R. A. I922. On the dominance ratio. Proc. Roy. Soc. Edin., 42, 32 I-34I.

FISHER, R. A. 1958. The Genetical Theory of Natural Selection. 2nd edition. Dover Publications, Inc., New York.

FYFE, J. L. I952. Plant breeding studies in leguminous forage crops. I. Natural cross-breeding in winter beans. F. Agric. Sci., 4I, 37 I-378.

HAYMAN, B. I. I953. Mixed selfing and random mating when homozygotes are at a disadvantage. Heredity, 7, 185-192. 
LEVENE, H., PAVLOVSKY, O., AND DOBZHANSKY, TH. I954. Interaction of the adaptive values in polymorphic experimental populations of Drosophila pseudo-obscura. Evolution, 8, 335-349.

MANDEL, S. P. H. I959a. The stability of a multiple allelic system. Heredity, I3, 289-302.

MANDEL, S. P. H. I959b. Stable equilibrium at a sex-linked locus. Nature, ${ }^{8} 8_{3}$, I $347-134^{8}$.

OWEN, A. R. G. 1953. A genetical system admitting of two distinct stable equilibria under natural selection. Heredity, 7, 97-102.

oWEN, A. R. G. I954. Balanced polymorphism of a multiple allelic series. Caryologia, supplement to vol. 6, I 240-1 $24 \mathrm{I}$.

owen, A. R. G. 1959. Mathematical Models for Selection in Natural Selection in Human Populations. Edited by D. F. Roberts and G. A. Harrison. Pp. I I-I6. Pergamon Press.

PARsons, P. A. I959a. Equilibria in auto-tetraploids under natural selection for a simplified model of viabilities. Biometrics, 15, 20-29.

PARSONS, P. A. 1959b. Some problems in inbreeding and random mating in tetrasomics. Agron. Four., 5I, 465-467.

TEISSIER, G., AND PH. L'HÉRITIER. I937. L'élimination des formes mutantes dans les populations de Drosophiles. 70ieme. Congres de Soc. Savantes, pp. 297-302.

TEISSIER, G. 1954. Conditions d'equilibre d'un couple d'alleles et superiorité des hétérozygotes. C.R. Acad. Sci., 238, 621-623.

\section{Note added in proof}

We have only recently found that Haldane (1957) has done a calculation similar to that at the beginning of section 2 .

HALDANE, J. B. s. I957. The conditions for co-adaptation in polymorphism for inversions. F. Genet., 55, $218-225$. 